\title{
Application of a custom-synthesized molecularly imprinted polymer for the selective isolation of total glucose and fructose from $100 \%$ fruit juice samples prior to instrumental analysis
}

https://doi.org/10.1515/molim-2018-0001

Received July 11, 2018; accepted July 12, 2018

\begin{abstract}
This article presents a novel sample preparation strategy that employed a custom-synthesized glucose fructose (G-F) specific molecularly imprinted polymer (MIP) powder as an adsorbent for the simultaneous and selective extraction, isolation and pre-concentration of total glucose and fructose from the complex and 'dirty` sample matrix of ' $100 \%$ ' fruit juices purchased from retail shops in Palapye, Botswana. The prepared G-F MIP powder demonstrated high selectivity, effective extraction and isolation for glucose and fructose from real samples of ' $100 \%$ ' fruit juice samples as evidenced by the calculated high extraction efficiencies (EEs) of over $90 \%$, with low percentage relative standard deviations (\%RSD) of below $7 \%$ for $n=6$, for both glucose and fructose when compared to the low EEs of below $25 \%$ by the non-imprinted polymer (NIP), regarded as the control. Furthermore, the G-F MIP showed lower selectivity towards the analogous molecules; maltose and lactose as supported by the low EEs of below $31 \%$. With the high affinity for glucose and fructose, the selective sample preparation strategy proposed herein presented itself as a potential procedure to be employed to improve the accurate analysis of adulterated artificial sugar sweeteners that are usually illegally added to the so-called `100\%` fruit juices by producers to improve their taste.
\end{abstract}

Keywords: `100\%` fruit juice, food adulteration, selective isolation, selective pre-concentration, imprinted polymers, selective sample preparation

\footnotetext{
*Corresponding author: Hawa W. Mukami,Botswana International University of Science and Technology, P.O Box private bag 16 Palapye, Botswana, E-mail: hawa.mukami@studentmail.biust.ac.bw Bareki S. Batlokwa, Botswana International University of Science and Technology, P.O Box private bag 16 Palapye, Botswana
}

\section{Introduction}

Premium quality 100\% fruit juices are on high demand due to their many important health benefits [1]. The continued high demand has made the juices command higher prices compared to other liquid refreshments. Furthermore, unscrupulous $100 \%$ fruit juice producers have resorted to the addition of trace concentrations of sweeteners in the form of artificial glucose and fructose that would not be easily detectable or even be easy to differentiate from the natural glucose and fructose present as part of the fruit. This practice is gaining popularity in order to keep up with the high demand and endeavors to improve the taste of the fruit juices.

The addition of the sugars is an illegal act and contributes to beverage adulteration [2]. This has given birth to a new crop of $100 \%$ fruit juices with a stinging sweet taste which are referred to as `100\%` fruit juices in this article.

The addition of the artificial sugars has necessitated the monitoring of fruit juice quality and authenticity. Sensitive analytical instruments such as isotope ratio mass spectrometer [3,4], high performance liquid chromatography mass spectrometer (HPLC-MS) [5], and enzymatic methods [6] areusually employed todifferentiate the adulterated artificial sugars from the natural sugars that form part of the natural fruit juices. The analytical instruments with their very high sensitivities possess the capability to accurately monitor adulteration in juice matrices even at trace levels; however, they have proved to be challenged by the 'dirty', complex matrix of the fruit juices which is often exacerbated by sample to sample variability of the fruits or fruit juices. The challenge is further compounded by the existence of analogous sugars in the matrix. Such matrices introduce severe interference in analytical separation steps. Additionally, very 'dirty' samples interfere with the sensitivity of detectors. Due 
to the complexity of juice matrices, samples often have to undergo targeted, selective and extensive sample preparation procedures such as selective sample isolation, pre-concentration and clean-up prior to instrumental analysis. In the past sample preparation strategies based on physical and chemical affinity of sugar molecules such as zeolite adsorption and activated carbon adsorption [7], have been employed to extract sugar molecules from sample matrices. These techniques, however, have the limitation of poor selectivity [8] which has led to research in the development of selective sorbents, characterized by high porosity, larger surface area and specific binding sites. One example of such selective sorbents is the molecularly imprinted polymers (MIPs) [9,10]. MIPs are highly crosslinked materials prepared from a prepolymerization complex (that is prepared from a template molecule and a functional monomer), and a cross-linker as a second monomer [11,12]. Subsequently, the template molecule is removed, revealing well-defined binding sites that correspond to the functionality, size and shape of the target molecules $[13,14]$. The selective binding ability of the MIPs to target analyte(s) is based on the theory that after polymerization [15], the MIPs go through a process of template (print molecule) removal which frees binding sites that conserve the interactions between the monomer(s) and the print molecule [16,17]. These interactions are responsible for the selective rebinding of target analytes [18]. According to H Pei et.al, MIPs have a broad range of applications from biological and chemical recognition $[19,20]$ to selective binding matrices in detection [21], separation [22] and purification [23,24]. In many cases they have been combined with solid phase extraction [25-28] and led to the advent of molecularly imprinted solid phase extraction (MI-SPE) technique which combines the advantages of SPE; low cost, speed, flexibility and the selectivity of MIPs. Molecular imprinting for glucose and fructose

This article presents preliminary results for the synthesis and evaluation of the effectiveness of a novel, synthesized glucose - fructose specific molecularly imprinted polymer (G-F MIP) as a MI-SPE sorbent for the simultaneous and selective extraction, isolation and preconcentration of total glucose and fructose from complex and 'dirty' sample matrix of ' $100 \%$ ' fruit juices prior to screening for illegally added glucose and fructose using analytical instruments.

\section{Methods}

\subsection{Materials and chemicals employed}

Tetrahydrofuran (THF), dimethyl sulfoxide ( $\mathrm{D}_{6}$-DMSO) were purchased from AppliChem (Darmstadt, Germany), methanol (99\%) purchased from Skylabs (Johannesburg, South Africa), acrylamide (99\%), 4,4'-azobis(4-cyano pentanoyl chloride) (ABCC), (98\%), ethylene glycol dimethacrylate EGDMA (99\%) were purchased from Sigma Aldrich ( Johannesburg, South Africa). D-fructose, D-glucose, maltose and lactose monohydrate were purchased from Sigma-Aldrich (Johannesburg South Africa). Ultra-pure water, was prepared by a Millipore-Q purification system from Merck, (Darmstadt, Germany) and $0.45 \mu \mathrm{m}$ pore sized ashless whatman filter papers were purchased from sigma-Aldrich (Johannesburg, South Africa)

\subsection{Instruments}

High performance liquid chromatography - refractive index detector (HPLC-RID), Agilent 1200 infinite series (LA, California, USA) was employed to determine the concentrations of the sugars. A scanning electron microscope (SEM) JSM-7100F purchased from JEOL (UK) Ltd (Welwyn Garden City, Hertfordshire) was used to obtain high resolved images of the synthesized polymers and assess their structural morphology. Thermo Scientific laboratory oven was purchased from Thermo Fisher Scientific Inc. (New York, USA), Benchmark hot plate was purchased from Benchmark Scientific (New Jersey, USA), micro-pipettes from BOECO (Berlin, Germany), a Tyler analytical balance from Mettler Toledo, A W.S Tyler ${ }^{\mathrm{TM}}$ (Johannesburg, South Africa), and a Laval stainless steel sieve $(45-200 \mu \mathrm{m})$ was purchased from Laval LAB (Minnesota, USA). Centrifuge, was purchased from VWR Catalyst (Philadelphia, PA, USA).

\subsection{HPLC-RID operating conditions}

The efficiency of the prepared MIPs throughout was investigated using HPLC-RID. Agilent Hi-plex Ca, $7.7 \times 300$ $\mathrm{mm}, 8 \mu \mathrm{m}$ column was employed for isocratic separation using $100 \%$ DI water as the mobile phase, and a flow rate of $0.5 \mathrm{~mL} / \mathrm{min}$. Column oven temperature of $80{ }^{\circ} \mathrm{C}$ was maintained. The injection volume was $20 \mu \mathrm{L}$. 


\subsection{Preparation of fructose and glucose imprinted polymer}

In a nitrogen purged reaction vessel, $0.736 \mathrm{mmol}$ of fructose and glucose, $10 \mathrm{mmol}$ of acrylammide, 41.2 mmol of EGDMA and $0.53 \mathrm{mmol}$ of ABCC, were added to a mixture of THF $(1.68 \mathrm{~mL})$ and DMSO $(21.02 \mathrm{~mL})$. The mixture was stirred at $900 \mathrm{rpm}$ for $45 \mathrm{mins}$. The reaction vessel was heated at $50^{\circ} \mathrm{C}$ and polymerization proceeded for $48 \mathrm{~h}$ under continuous stirring. The resulting polymer monolith was finely ground and dried in an oven at $50^{\circ} \mathrm{C}$ for $4 \mathrm{~h}$. The resultant polymer powder was then washed twice employing ultra-pure water to remove unreacted materials and dried at $50^{\circ} \mathrm{C}$. The powder was sieved to obtain homogeneous particles of $<50 \mu \mathrm{m}$. The templates (fructose and glucose) were exhaustively removed from the imprinted polymer by washing severally in $99 \%$ methanol. After every $5 \mathrm{~h}$ washing cycle, the MIP powder was separated from the used methanol by filtration and fresh methanol was then added to further remove the templates. After every cycle, the concentrations of fructose and glucose in the filtrates were determined employing HPLC-RID until a point where the concentrations remained the same, thus marking optimal template removal. The resultant MIP powder particles were then recovered through filtration and then dried at $50^{\circ} \mathrm{C}$. The voltage values from the HPLC-RID that correlated to concentrations were then plotted against the number of washing cycles. A control polymer, non-imprinted polymer (NIPs) was also prepared employing a similar procedure except that the template molecules were absent during preparation. The resultant NIP powder was then subjected to all procedures that were performed on the MIP powder.

\subsection{Characterization of G-F MIP and NIP powders}

\subsubsection{SEM Characterization}

The size homogeneity of the polymer particles was first achieved through the use of standard sieves. SEM was employed to further characterize size, geometry and surface morphological information of the MIPs and NIPs. The powders were carbon-coated under a polaron range high vacuum pressure sputter coater and placed on a $1 \mathrm{~cm}$ tall sample holder. The operation was performed under high vacuum and beam acceleration voltage of $10 \mathrm{kV}$.

\subsection{Batch rebinding experiments}

Inorder to evaluate the binding capability of the prepared MIP, batch rebinding experiments were performed, after which the HPLC-RID was employed to determine the concentrations of glucose and fructose before and after application of the prepared MIPs. The percentage extraction efficiencies of the MIPs were calculated following equation 1 below.

$$
\begin{aligned}
& \% \text { Extraction } \\
& \text { efficiency }
\end{aligned}=\frac{\text { Concentration before employing MIP- Concentration after employing MIP }}{\text { Concentration before employing MIP }} \times 100
$$

For the $\mathrm{pH}$, the real juice sample $\mathrm{pH}$ was obtained as $\mathrm{pH}$ 3.5 , this $\mathrm{pH}$ value was employed in all the analysis. All experiments were performed in triplicates $(n=3)$.

\subsubsection{Optimization of quantity of MIP powder needed for maximum glucose and fructose extraction}

Increasing quantities of MIP powder from $0 \mathrm{mg}$ at intervals of $10 \mathrm{mg}$ were added to $30 \mathrm{~mL}$ of $20 \mathrm{mg} / \mathrm{L}$ equimolar standard solutions of glucose and fructose. The solution mixtures with different quantities of MIPs were left overnight for equilibration and maximum extraction. The MIP powders were filtered off and the concentrations of the glucose and fructose in the filtrate before and after employing the MIP powders were determined using the HPLC-RID and calculated via equation 1 . The experiment was repeated with increasing quantities of the MIP powder until a point where the calculated extraction efficiency remained constant with increasing quantity, thus marking the optimal quantity needed for maximum extraction. A plot of \% extraction efficiency versus quantity of G-F MIP powder added was constructed.

\subsubsection{Optimization of Time needed for maximum fructose and glucose extraction}

Using the optimized MIP quantity in (2.6.1), optimal time needed for the MIP to remove maximum glucose and fructose from standard glucose-fructose solutions was investigated. The optimized quantity of the G-F MIP powder was added to various $30 \mathrm{~mL}$ of $20 \mathrm{mg} / \mathrm{L}$ equimolar concentrations of glucose and fructose standard solutions for an increasing duration at $10 \mathrm{~min}$ intervals starting from $0 \mathrm{~min}$. Each mixture was left to equilibrate at room temperature. The experiment was repeated with increasing time until a point where the calculated \% extraction efficiency for the different times 
remained constant with increased time, thus marking the optimal time needed for maximum extraction. A plot of $\%$ extraction efficiency against time needed for maximum extraction was constructed.

\subsubsection{Selectivity studies}

To evaluate the ability of the prepared MIP powder to favorably extract the target analytes (glucose and fructose), analogous molecules; lactose and maltose were chosen to compete with the target analytes via rebinding experiments described in section 2.4. An optimal quantity of MIP powder was added to $30 \mathrm{~mL}$ of $20 \mathrm{mg} / \mathrm{mL}$ equimolar concentrations of glucose, fructose, lactose and maltose and allowed to equilibrate for optimal time. The MIP powder was filtered off and the concentrations of glucose, fructose, maltose and lactose in the filtrate obtained after equilibration under optimal conditions were analysed employing HPLC-RID and thereafter the extraction efficiencies by the prepared MIP for each analyte were calculated following equation 1.

Similarly, the same procedure was followed employing the NIP powder that was employed as the control and the extraction efficiencies for each analyte were calculated following equation 1 .

\subsubsection{Method Validation}

\subsubsection{Linearity}

Linearity of the method was investigated through triplicate injections of spiked ' $100 \%$ ' fruit juice at different glucose and fructose equimolar concentrations ranging from 0 to $50 \mathrm{mg} / \mathrm{L}$. Calibration curves of concentration verses peak areas for glucose and fructose were plotted and the correlation coefficient $\left(R^{2}\right)$ which is a measure of linearity was then obtained from the linear plots of each of the sugars respectively.

\subsubsection{Detection Limits}

The Limits of detection (LODs) and Limits of quantification (LOQs) were determined by employing a method based on the analytical curve parameters according to equations 2 and 3.

$$
\begin{aligned}
& \angle O D=\frac{(\mathrm{SD} \times 3.3)}{\mathrm{m}} \\
& \angle O Q=\frac{(\mathrm{SD} \times 10)}{\mathrm{m}}
\end{aligned}
$$

Where $\mathrm{m}$ is the slope and SD is the standard deviation.

\subsubsection{Application of method to real samples}

$' 100 \%$ ' apple fruit juice samples were diluted with distilled water (dilution factor 500). The samples were then filtered using $0.45 \mu \mathrm{m}$ pore sized filter paper and the concentrations of glucose and fructose before and after application of G-F MIP in the filtrates were obtained employing HPLC-RID. Chromatograms of glucose and fructose concentration of the filtrates before and after employing the G-F MIP were obtained and the enrichment factor (EF) calculated using equation 4;

$$
E F=\frac{\mathrm{a}}{\mathrm{b}}
$$

Where:

$E F$ is enrichment factor,

$a$ is glucose or fructose concentration obtained after filtering and application of G-F MIP and

$b$ is glucose or fructose concentration obtained after filtering.

Precision was also expressed as a percentage relative standard deviation (\% RSD) for $\mathrm{n}=6$ and was calculated following equation 5. Precision may be expressed as repeatability and it's a measure of the degree of conformity between independent measurement results acquired under set 198 conditions.

$$
\% R S D=\frac{\text { standard deviation }}{\text { mean }} \times 100
$$

\section{Results and Discussion}

\subsection{Synthesis of Fructose-glucose MIP}

It is imperative that the template molecules be removed successfully for optimum performance of MIPs as adsorbents [29). For this, the MIP was extensively washed until there was no further observable changes in the voltage values (equivalence of the concentrations of the templates) obtained despite further washing with fresh solvent as marked by a plateau from the $5^{\text {th }}-10^{\text {th }}$ wash in Figure 1. The plateau indicates that there was no significant statistical change thus marking optimal template removal. It can be observed that after 10 subsequent interval washes, satisfactory amounts of glucose and fructose molecules were removed from the MIP. The plateau confirmed optimal template removal by the method employed. Optimal template removal is necessary as it is the one that frees reaction sites (cavities) for the subsequent rebinding of the target analytes. 
Furthermore, optimal template removal is vital for the elimination of template bleeding which leads to erroneous results (false positives).

\subsection{Morphology of the synthesized polymer particles}

SEM micrographs of the MIPs and NIPs obtained did not show notable variations, hence, only the SEM micrograph of the MIPs was reported (see Figure 2). With the help of standard sieves particle size homogeneity was achieved and the size of the MIP particles was estimated to be $<20$ $\mu \mathrm{m}$ as per the scale on Figure 2, which is a size small enough to be associated with increased surface area resulting in enhanced sorbent capacity. The smaller the particle size the higher the surface area and sorbent capacity [30]. The image revealed that the MIP particles were spherical, which Mayes et al and Mosbach et al reported to be a good geometry for sorbent materials [31,32]. The surface of the particles seemed to be rough and porous which is an excellent characteristic for adsorption [33].

\subsection{Batch Rebinding Experiments}

\subsubsection{Optimization of MIP powder needed for maximum glucose and fructose extraction}

Optimization of sorbent mass is important to avoid wasting of sorbent by adding excess unnecessarily or adding low sorbent mass thus compromising the results [25]. For this work, the optimum MIP powder needed for maximum extraction was found to be $60 \mathrm{mg}$, marked by a point at which the plateau starts to form on the plot of percentage extraction efficiency versus mass of sorbent (see Figure 3). Increase in sorbent mass from 10 $\mathrm{mg}$ to $60 \mathrm{mg}$ resulted in significant adsorption, which was attributed to the parallel increase in binding sites. However, it was observed that after $60 \mathrm{mg}$ there was no further change in percentage extraction which marked the saturation point of the binding sites. Therefore $60 \mathrm{mg}$ was employed in this study.

The optimized mass was used to determine adsorption capacity following equation 6 below. Adsorption capacity for the prepared MIP was obtained as $9.30 \mathrm{mg} / \mathrm{g}$ and $9.07 \mathrm{mg} / \mathrm{g}$ for glucose and fructose respectively.

$$
q=\frac{[\mathrm{Ci}-\mathrm{Cf}] \times \mathrm{V}}{\mathrm{W}}
$$

Where, $q$ is the adsorption capacity, $C i$ and $C f$ are the initial and final concentrations respectively, $\mathrm{V}$ is the volume of sample employed and $\mathrm{W}$ is the mass of the sorbent. Parmpi et al reported binding capacities

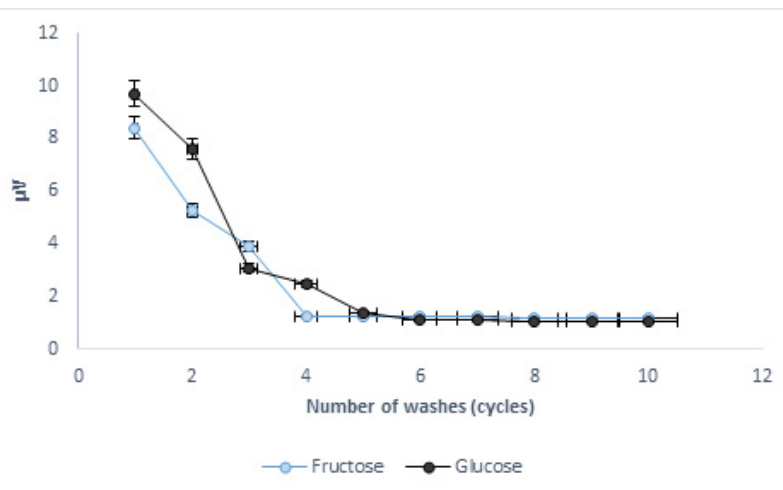

Figure 1: A voltage plot confirming the removal of glucose and fructose templates from MIPs

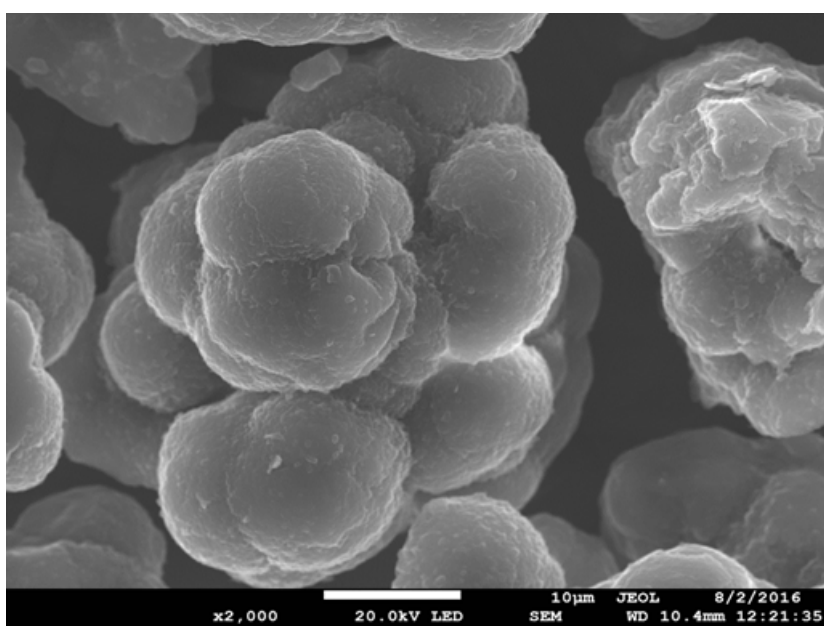

Figure 2: SEM image of MIP particles

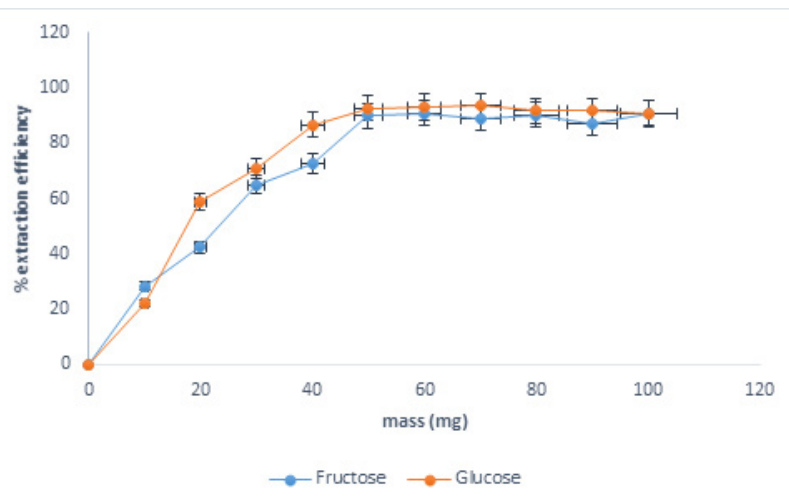

Figure 3: Optimization of the maximum amount of MIP required for optimal removal efficiency

of $7.15 \mathrm{mg} / \mathrm{g}$ for fructose and glucose. In their study, the authors findings have shown that the proposed MIP gels selectively bound glucose and fructose analytes in a water swollen state [34]. Rajagpal et al also reported a binding capacity of about $40 \mathrm{nmol} / \mathrm{mg}$ which signified an effective binding pocket [5]. 


\subsubsection{Optimization of time needed for maximum fructose and glucose extraction}

Sufficient time was required for both glucose and fructose analytes to rebind to the sites. This is since binding sites (cavities) are sometimes not on the surface, due to the type of polymerization employed in this study, bulk polymerization, which results in bulk particles having binding sites that are embedded inside. Thus, time had to be optimized for equilibration. The optimal time was arrived at 20 min marked by a point at which the graph plateaued (see Figure 4). Percentage extraction of the glucose and fructose analytes increased with increase in contact time from $0 \mathrm{~min}$ up to $20 \mathrm{~min}$, after which the rate of adsorption was relatively uniform. The linear increase observed from time $0 \mathrm{~min}$ to $20 \mathrm{~min}$, resulted from the increase in interaction time between the MIP and the analyte molecules which increased the chances of adsorption [35]. In a study carried out by Rajkumar et al on fructose recognition by imprinted polymers an optimum time of 2 hours was reported [5]. This compares with the current work findings very well, in that, the prepared G-F MIP has a relatively lower equilibration time.

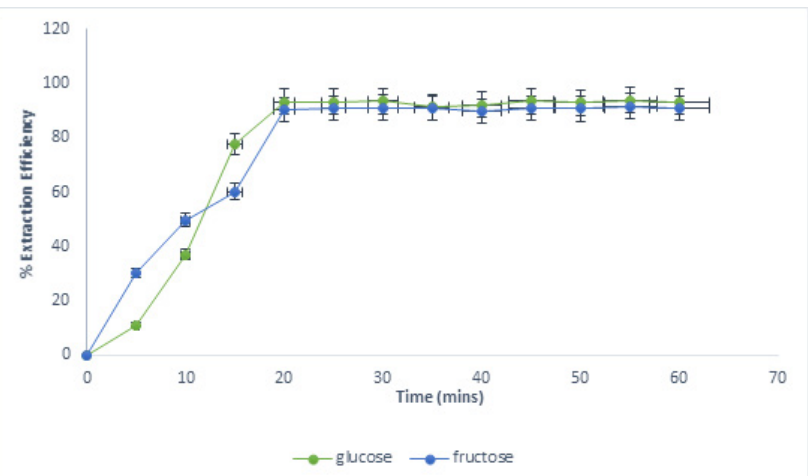

Figure 4: Optimization of time required for maximum removal of fructose and glucose

\subsection{Selectivity studies}

MIP efficiency cannot solely be evaluated on the ability of the polymer to rebind the analyte(s) [36], but also on its discriminative ability towards target analytes in the presence of analogous molecules [36,37]. Following Figure 5, the percentage extraction efficiencies by the MIP powder of glucose and fructose was observed to be much higher than those of lactose and maltose. These observations were an indication that the binding sites in the MIP had a higher affinity for glucose and fructose. In addition, the extraction efficiency of the G-F MIP was observed to be superior in extracting glucose and fructose when compared to the NIP in the same environment. The MIP bound a higher percentage of $92.45 \%$ and $93.12 \%$ for glucose and fructose respectively compared to the much lower percentages of $23.6 \%$ and $31.4 \%$ for maltose and lactose respectively. This was attributed to the binding sites that were freed during template removal and left a memory for the target analytes to rebind. The NIP on the other hand showed non selectivity and lower performance than the prepared G-F MIP as demonstrated by statistically the same percentage extraction efficiencies that had a low range of $(22.7 \%-33.4 \%)$ for both the target and the analogous molecules (see Figure 5). The non-selectivity of the NIP is attributed to the lack of inclusion of the templates during the synthesis process.

\subsection{Method validation}

\subsubsection{Linearity}

Table 1 summarizes the linearity of the calibration curves for glucose and fructose in the form of correlation coefficients of 0.9959 and 0.9945 for glucose and fructose respectively. $R^{2}$ values $>0.995$ are considered to show a good linearity of the data obtained, therefore the obtained correlation

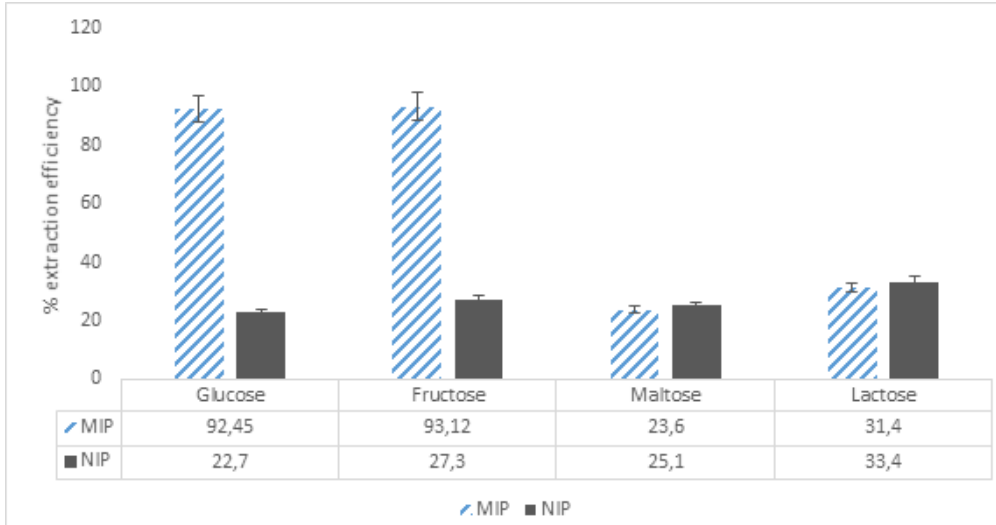

Figure 5: percentage extraction efficiency of sugars by employing both the MIPs and NIPs adsorbents. 
coefficient in this study were found to be statistically acceptable [38]. The results proved that there was a fairly strong linear correlation between the sample concentrations of glucose and fructose and the corresponding determined chromatographic peak areas or response.

\subsubsection{LODs and LOQs}

The calculate LODs and LOQs were obtained and found to have a low range of $3.975-7.185 \mathrm{mg} / \mathrm{L}$ and $12.04-21.77$ $\mathrm{mg} / \mathrm{L}$ respectively. These LODs and LOQs indicated that the method was more sensitive compared to the relatively high LOD and LOQ values of major sugars in apple juice reported by Zielinski et al. which ranged from 7.56 - 56.86 $\mathrm{mg} / \mathrm{L}$ and 25.21-192.88 mg/L respectively for all sugars including glucose and fructose [39].

\subsubsection{Application of method in real samples}

A close study and evaluation of the peak intensities obtained in Figure 6, of before and after G-F MIP application to $100 \%$ juice samples showed an increase from $8.5 \mathrm{mV}$ to $15.8 \mathrm{mV}$ for glucose and $15.9 \mathrm{mV}$ to $31.9 \mathrm{mV}$ for fructose. This marked a 2-fold enrichment factors (EFs) or pre-concentration factors for glucose and fructose. The EFs were calculated following equation 4.

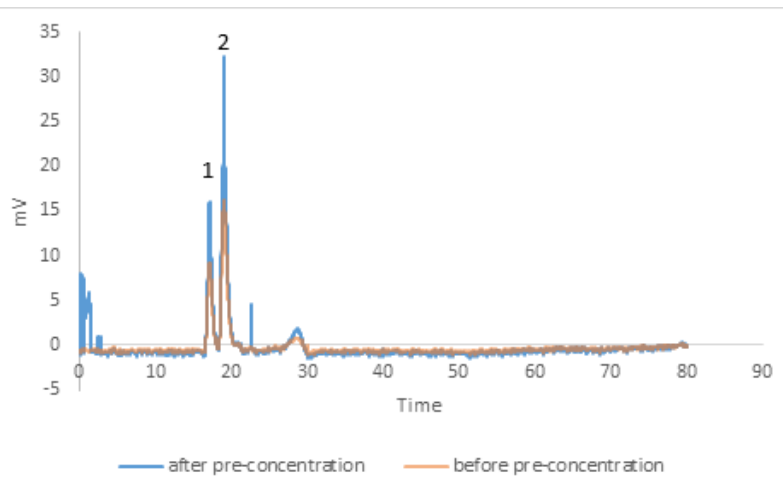

Figure 6: chromatograms of juice samples, with concentrations of (1) glucose and (2) fructose before and after MIP application

Relatively high extraction efficiencies of more than $90 \%$ and low RSDs of less than $7 \%$ for $n=6$ for both glucose and fructose were recorded and demonstrated fairly good efficiency of the G-F MIP.

\section{Conclusion}

This paper presented the successful synthesis of a G-F MIP that exhibited excellent affinity and specificity towards fructose and glucose by demonstrating success in selectively binding a higher percentage (> 90\% EEs) for the analyte molecules (glucose and fructose), than the analogous molecules, maltose and lactose $(<34 \%$ EEs) in

Table 1: Linear regression parameters obtained from standard calibration curve for a $100 \%$ apple juice sample spiked with various concentrations of glucose and fructose

\begin{tabular}{lll}
\hline Sugar & Regression Equation & Regression coefficient (r2) \\
\hline D-Fructose & $y=6079.4 x-439.8$ & 0.9945 \\
D-glucose & $y=2233 x-153.8$ & 0.9959 \\
\hline
\end{tabular}

Table 2: Extraction efficiencies of glucose and fructose with the G-F MIP and associated \% RSD calculated at three spiked equimolar concentrations of glucose and fructose

\begin{tabular}{lrrrr}
\hline Spiking concentration $\mathrm{mg} / \mathrm{L}$ & \multicolumn{2}{c}{ \% Extraction Efficiencies (EEs) } & \multicolumn{2}{c}{ \% RSD } \\
& \multicolumn{2}{c}{ Glucose Fructose } & \multicolumn{2}{c}{ Glucose Fructose } \\
\hline 5 & 93.5 & 91.7 & 6.13 & 4.89 \\
15 & 90.8 & 92.2 & 4.78 & 5.94 \\
25 & 92.1 & 92.6 & 5.97 & 5.13 \\
\hline
\end{tabular}

Table 3: Limits of detection and quantification

\begin{tabular}{lll}
\hline Sugar & LOD mg/L & LOQ mg/L \\
\hline D-Glucose & 7.2 & 21.8 \\
D-Fructose & 4.0 & 12.0 \\
\hline
\end{tabular}


competitive binding environments. The study presented herein demonstrated that extraction efficiencies of glucose and fructose (> 90\% EEs) employing G-F MIP were significantly higher than those obtained after employing the NIP, control polymer ( $<28 \%$ EEs). Approximately 2-fold enrichment factors were achieved when the method was applied to selectively extract and pre-concentrate glucose and fructose from 100\% apple juice samples simultaneously. Thus, the novel G-F MIP prepared in this work is the first attempt in dual imprinting of both glucose and fructose template molecules. The G-F MIP presented itself as a potential effective sorbent that can be employed for the selective extraction, isolation and pre-concentration of glucose and fructose from 'dirty' complex matrices prior to accurate analysis by sensitive analytical instruments.

Acknowledgement: Botswana International University of Science and Technology (BIUST) is acknowledged for availing the resources required for the success of this research.

\section{References}

[1] Clemens R, Drewnowski A, Ferruzzi MG, Toner CD, Welland D. Squeezing fact from fiction about $100 \%$ fruit juice. Adv Nutr [Internet]. 2015;6(2):236S-243S. Available from: http:// advances.nutrition.org/content/6/2/236S.abstract

[2] Magdas DA, Cristea G, Puscas R, Tusa F. The use of isotope ratios in commercial fruit juices authentication. Rom J Phys. 2014;59(3-4):355-9.

[3] Figueira R, Gastoni W, Filho V, Ducatti C, Milene A, Nogueira P. Isotope analysis ( $\delta 13 \mathrm{C}$ ) of pulpy whole apple juice. Cienc e Tecnol Aliment. 2011;31(3):660-5.

[4] Kandimalla VB, Ju H. Molecular imprinting: A dynamic technique for diverse applications in analytical chemistry. Anal Bioanal Chem. 2004;380(4):587-605.

[5] Rajkumar R, Warsinke A, Mohwald H, Scheller F.W, Katterle $M$. Analysis of recognition of fructose by imprinted polymers. Talanta. 2008;76:1119-23.

[6] Mousavi S.M, Moghadam M.T. Separation of Sugar from Molasses by Ultrafiltration and Nanofiltration. world Appl Sci J. 2009;7(5):632-6.

[7] Clean Air Technology EPA. Choosing an adsorption system for VOC: carbon, zeolite or polymers? Catc Tech Bull [Internet]. 1999;(May):EPA-456/F-99-004. Available from: http://www. epa.gov/ttn/catc

[8] Knaebel K.S. Adorbent selection. Albright's Chem Eng Handb [Internet]. 2008;20:1119-71. Available from: https://books. google.com/books?id=HYB3Udjx_FYC\&pgis $=1$

[9] Yan H, Ho Row K. Characteristic and Synthetic Approach of Molecularly Imprinted Polymer. Int J Mol Sci [Internet]. 2006;7:155-78. Available from: www.mdpi.org/ijms/

[10] Deng F, Li Y, Luo X, Yang L, Tu X. Preparation of conductive polypyrrole/TiO 2 nanocomposite via surface molecular imprinting technique and its photocatalytic activity under simulated solar light irradiation. Colloids Surfaces A Physicochem Eng Asp [Internet]. 2012;395:183-9. Available from: http://dx.doi.org/10.1016/j.colsurfa.2011.12.029

[11] Vasapollo G, Sole R Del, Mergola L, Maria Rosaria Lazzoi, Scardino A, Scorrano S, Mele, G. Molecularly imprinted polymers: Present and future prospective. Int J Mol Sci. 2011;12(9):5908-45.

[12] Ramstram 0, Ansell R.J. Molecular imprinting technology: Challenges and prospects for the future. Chirality. 1998;10(3):195-209.

[13] Lok C.M, Son R. Application of molecularly imprinted polymers in food sample analysis - A perspective. Int Food Res J. 2009;16(2):127-40.

[14] Stevens M.G.F, Batlokwa B.S. Environmentally Friendly and Cheap Removal of Lead (II) and Zinc (II) from Wastewater with Fish Scales Waste Remains. Int J Chem [Internet]. 2017;9(4):22. Available from: http://www.ccsenet.org/journal/index.php/ijc/ article/view/68378

[15] Nikesh B. Samarth, Vinayak Kamble, Prakash A. Mahanwar AVR and AVK. A historical perspective and the development of molecular imprinting polymer-A review. Chem Int. 2015;4(4):202-10.

[16] Garcia R, Cabrita M.J, Maria A, Freitas C. Application of Molecularly Imprinted Polymers for the Analysis of Pesticide Residues in Food-A Highly Selective and Innovative Approach. Am J Anal Chem. 2011;2(December):16-25.

[17] Shekarchizadeh H, Ensa A.A, Kadivar M. Selective determination of sucrose based on electropolymerized molecularly imprinted polymer modi fi ed multiwall carbon nanotubes / glassy carbon electrode. Ecol Chem Eng. 2013;33:3553-61.

[18] Öpik A, Menaker A, Reut J, Syritski V. Molecularly imprinted polymers: A new approach to the preparation of Functional materials. Proc Est Acad Sci. 2009;58(1):3-11.

[19] Xu L, Huang Y-A, Zhu Q-J, Ye C. Chitosan in MolecularlyImprinted Polymers: Current and Future Prospects. Int I Mol Sci [Internet]. 2015;16(8):18328-47. Available from: 10.3390/ ijms160818328\%5Cnhttp://search.ebscohost.com/login. aspx?direct $=$ true $\& d b=a 9 h \& A N=109123181 \&$ site $=$ ehost - live

[20] Janeba D. Molecular simulations of. ESCEC. 2007;197-204.

[21] Ye L, Mosbach K. Molecularly imprinted microspheres as antibody binding mimics. React Funct Polym. 2001;48(13):149-57.

[22] Scheller F.W, Yarman A. Biochemistry \& Analytical Biochemsitry Bio vs . Mimetics in Bioanalysis : An Editorial. Biochem Anal Biochem. 2015;4(2).

[23] Zhao M, Shen G. Application of molecularly imprinted polymers. ICAMEM 2013 Singapore. 2015;(3):87-9.

[24] Carro-Diaz A.M, Lorenzo-Ferreira RA. Molecularly imprinted polymers for sample preparation: A review. Anal Chim Acta. 2010;668(2):87-99.

[25] Qiao F, Sun H, Yan H, Row K.H. Molecularly Imprinted Polymers for Solid Phase Extraction. Chromatographia. 2006;64(1112):625-34.

[26] Yi L-X, Fang R, Chen G-H. Molecularly Imprinted Solid-Phase Extraction in the Analysis of Agrochemicals. J Chromatogr Sci [Internet]. 2013;51(7):608-18. Available from: https:// academic.oup.com/chromsci/article-lookup/doi/10.1093/ chromsci/bmt024 
[27] Olcer Y.A, Demirkurt M, Demir M.M, Eroglu A.E. Development of molecularly imprinted polymers (MIPs) as a solid phase extraction (SPE) sorbent for the determination of ibuprofen in water. RSC Adv [Internet]. 2017;7(50):31441-7. Available from: http://xlink.rsc.org/?DOI=C7RA05254E

[28] C. Widstrand, H. Bjork E.Y. Chem Technol. 2006;(June):22-3.

[29] Haupt K. Peer Reviewed: Molecularly Imprinted Polymers: The Next Generation. Anal Chem. 2003;75(17):376 A-383 A.

[30] Stevens MGF, Batlokwa BS. Multi-templated Pb-Zn-Hg Ion Imprinted Polymer for the Selective and Simultaneous Removal of Toxic Metallic Ions from Wastewater. Int J Chem [Internet]. 2017;9(2):1-10. Available from: http://www.ccsenet.org/ journal/index.php/ijc/article/view/65613

[31] Mayes a G, Mosbach K. Molecularly imprinted polymer beads: suspension polymerization using a liquid perfluorocarbon as the dispersing phase. Anal Chem. 1996;68(21):3769-74.

[32] Mosbach K, Haupt K. Some new developments and challenges in non-covalent molecular imprinting technology. J Mol Recognit [Internet]. 1998;11(1-6):62-8. Available from: http://doi.wiley.com/10.1002/\%28SICI\%291099$1352 \% 28199812 \% 2911 \% 3 \mathrm{~A} 1 / 6 \% 3 \mathrm{C} 62 \% 3$ A\%3AAID-JMR391\%3E3.0.CO\%3B2-5

[33] Liu B, Cang H, Jin J. Molecularly imprinted polymers based electrochemical sensor for 2,4-dichlorophenol determination. Polymers (Basel). 2016;8(8).
[34] Parmpi P, Kofinas P. Biomimetic glucose recognition using molecularly imprinted polymer hydrogels. Biomaterials. 2004;25(10):1969-73.

[35] Batlokwa BS, Mokgadi J, Majors R, Turner C, Torto N. A novel molecularly imprinted polymer for the selective removal of chlorophyll from heavily pigmented green plant extracts prior to instrumental analysis. J Chem. 2013;2013.

[36] Roland RM, Bhawani SA. Synthesis and Characterization of Molecular Imprinting Polymer Microspheres of Piperine: Extraction of Piperine from Spiked Urine. J Anal Methods Chem [Internet]. 2016;2016:1-6. Available from: https://www. hindawi.com/journals/jamc/2016/5671507/

[37] Koohpaei AR, Shahtaheri SJ, Ganjali MR, Forushani AR, Golbabaei F. Application of multivariate analysis to the screening of molecularly imprinted polymers (MIPs) for ametryn. Talanta. 2008;75(4):978-86.

[38] Mothibedi K, Mokgadi J, Torto N. Determination of Flavonoids in Ginkgo Biloba Using Bond Elut Plexa Solid Phase Extraction Sorbent for Cleanup and HPLC-DAD Analysis. :0-5.

[39] Zielinski AAF, Braga CM, Demiate IM, Beltrame FL, Nogueira A, Wosiacki G. Development and optimization of a HPLC-RI method for the determination of major sugars in apple juice and evaluation of the effect of the ripening stage. Food Sci Technol [Internet]. 2014;34(1):38-43. Available from: http:// www.scielo.br/scielo.php?script=sci_arttext\&pid=S0101$20612014000100005 \& \operatorname{lng}=e n \& n r m=i s o \& \operatorname{lng}=e n$ 